\title{
Targeting the neurokinin-1 receptor inhibits growth of human colon cancer cells
}

\author{
AGNÈS GARNIER $^{1}$, JODY VYKOUKAL $^{2}$, JOCHEN HUBERTUS $^{1}$, ECKHARD ALT $^{3}$, \\ DIETRICH VON SCHWEINITZ ${ }^{1}$, ROLAND KAPPLER ${ }^{1}$, MICHAEL BERGER $^{1}$ and MATTHIAS ILMER ${ }^{2,4}$

\begin{abstract}
${ }^{1}$ Department of Pediatric Surgery, Research Laboratories, Dr von Hauner Children's Hospital, Ludwig Maximilians University Munich, Munich, Germany; ${ }^{2}$ Department of Translational Molecular Pathology, The University of Texas M.D. Anderson Cancer Center, Houston, TX; ${ }^{3}$ Department of Medicine, Vascular and Thoracic Surgery, Hospital of the Ludwig Maximilians University Munich, Munich, Germany
\end{abstract} \\ Tulane University Health Science Center, New Orleans, LA, USA; ${ }^{4}$ Department of General, Visceral, Transplantation,
}

Received March 9, 2015; Accepted April 15, 2015

DOI: 10.3892/ijo.2015.3016

\begin{abstract}
The substance P(SP)/neurokinin-1 receptor (NK1R) complex and the Wnt cascade are pivotal signaling pathways in the regulation of cell growth and hence, potent targets for future anticancer therapies. However, while the Wnt cascade has long been associated with colon cancer, little is known about the expression of the NK1R complex as a potential target in this tumor and its molecular basis in tumorigenesis in general. We treated the human colon cancer cell lines LiM6 and DLD1 with the NK1R antagonist and the clinical drug aprepitant (AP) and analyzed both growth response and downstream mechanisms using MTT-assay, reverse phase protein array (RPPA), western blot, Super TOP/FOP, confocal microscopy, and sphere formation ability (SFA) assays. Following NK1R blockage, we found significant growth inhibition of both colon cancer cell lines. When analyzing downstream mechanisms, we found a striking inhibition of the canonical Wnt pathway represented by decreased Super TOP/FOP and increased membrane stabilization of $\beta$-catenin. This effect was independent from baseline Wnt activity and mutational status of $\beta$-catenin. Further, treatment of colon cancer cells grown under cancer stem cell (CSC) conditions reduced sphere formation in both number and size after a single treatment period. We show that the NK1R can be a potent anticancer target in colon cancer and that NK1R antagonists could potentially serve as future anticancer drugs. This effect was seen not only in primary cancer cells but, for the first time, also in CSC-like cells, potentially including these cells in a therapeutic effect. Also, we describe the robust inhibition
\end{abstract}

Correspondence to: Dr Matthias Ilmer, Department of General, Visceral, Transplantation, Vascular and Thoracic Surgery, Hospital of the University of Munich, Ludwig Maximilians University Munich, Marchioninistreet 15, D-81377 Munich, Germany

E-mail: matthias.ilmer@googlemail.com

Key words: neurokinin-1 receptor, NK1R-antagonists, substance P, Wnt signaling, $\beta$-catenin, colon cancer of canonical Wnt signaling through targeting the SP/NK1R signaling cascade. These findings give important insight into the molecular mechanisms of the SP/NK1R complex as a critical component in tumorigenesis and could help to identify future anticancer therapies for colon and other Wnt-activated cancers.

\section{Introduction}

Colorectal cancer (CRC) is the third most common cancer in the world with an incidence of 9.4 and $10.1 \%$ in men and women, respectively. Although recent progress in chemotherapy regimen has increased survival significantly, it remains a major cause of mortality and morbidity (1).

Different molecular alterations have been described in CRC such as mutation of the oncogene KRAS or the tumor suppressor p53 as well as mutation in the TGF- $\beta$ pathway (2). However, $90 \%$ of CRC arise from an activating mutation of the canonical Wnt pathway. Particularly, mutation of the adenomatous polyposis coli (APC) gene is believed to be an initiating event in $80 \%$ of sporadic colorectal tumors but is also responsible for familial adenomatous polyposis (3). This mutation induces the stabilization of $\beta$-catenin resulting in its accumulation in the nucleus, where it complexes the DNA-binding family TCF/LEF to activate the transcription of Wnt target genes such as MYC, CCND1 and AXIN2. Overexpression of Wnt target genes has been associated with chromosome instability and tumorigenesis (4). This constant activation of Wnt signaling then leads to colorectal neoplasia with aberrant crypt foci especially in the early stages of tumor formation (5).

Further, high $\beta$-catenin/Wnt signaling was previously linked to cancer stem cell (CSC) properties in colorectal cancer (6) and pancreatic ductal adenocarcinoma (7). CSCs are a rare population of cancer cells exhibiting stem cell traits, such as self-renewal and tumorigenesis. These stem cells often reside in a particular niche, have higher resistance to chemotherapy as well as radiotherapy, and are also capable of invading and migrating to other tissues $(8,9)$. Therefore, besides the initiation of the primary tumor, CSCs have been associated with 
metastasis formation and cancer relapse (10). Within this context, targeting Wnt signaling is one of the most promising approaches and several studies have identified Wnt as a potent anticancer target in CRC (6-9). However, the main problem resides today in the difficulty to produce clinical drugs that effectively target this pathway in cancer cells.

Independently from this, substance P (SP) is an undecapeptide of the tachykinin family. It is widely distributed throughout the body and has a wide variety of functions under physiological conditions (11-13). It binds to the neurokinin (NK) receptor (NKR) family (NK1R, NK2R and NK3R), but preferentially to NK1R for which it has a high affinity, and regulates many biological functions mainly in the central and peripheral nervous system (14-16). Recently, it has been reported that the $\mathrm{SP} / \mathrm{NK} 1 \mathrm{R}$ complex is part of the tumor microenvironment and is involved in tumor cell proliferation, angiogenesis and migration (14). The small molecule aprepitant (AP) is known to cause a variety of therapeutic effects in humans, partially depending on the dosage in which it is used. In low doses, AP reduces nausea and vomiting induced by chemotherapy. Of note, for this indication, AP is approved by the Food and Drug Administration (FDA). There are currently ongoing clinical trials investigating the potential to reduce nausea and vomiting in postoperative patients, including children. In medium doses, AP has been shown to relieve symptoms of depression $(17,18)$. In experimental in vitro and in vivo settings, higher doses of NK1R antagonists induce robust inhibition of tumor growth and apoptosis in cancer cells of a great variety of adult and childhood cancers, including colon cancer (19-21). However, little is known about the intracellular signaling responsible for the effects. Here, we sought to investigate the underlying molecular mechanisms in two CRC cell lines. We found a striking inhibition of canonical Wnt signaling upon treatment with AP, resulting in potent growth inhibition. Therefore, our discoveries could have important implementations for the introduction of new innovative anticancer strategies against colon cancer.

\section{Materials and methods}

Cell culture. We used two colorectal cell lines; DLD1 was obtained from ATCC (American Type Culture Collection) and LiM6, a derivative of the LS174T cell line, was a kind gift of Dr Robert S. Bresalier (MDACC) (22). All cell lines were grown in RPMI-1640 medium (Invitrogen, Carlsbad, CA, USA) supplemented with $10 \%$ FCS (Invitrogen) and $1 \%$ penicillin/streptomycin (Invitrogen) at $37^{\circ} \mathrm{C}$ in a humidified incubator with $5 \% \mathrm{CO}_{2}$.

Drugs. Aprepitant (AP), an NK1R antagonist and substance P (SP), an NK1R agonist were purchased from Selleck Chemicals and Sigma-Aldrich and were dissolved in DMSO and $0.1 \mathrm{M}$ acetic acid, respectively.

Proliferation assays. Cell proliferation was assessed using 1-(4,5-dimethylthiazol-2-yl)-3,5-diphenylformazan (MTT) assay. Ten thousand cells/well were seeded into 96-well plates (NUNC, Langenselbold, Germany). After $24 \mathrm{~h}$, cells were treated with increasing doses of AP for $48 \mathrm{~h}$ or with DMSO. To assess cell viability, a final concentration of $0.5 \mathrm{mg} / \mathrm{ml}$
(MTT formazan powder, Sigma-Aldrich diluted in PBS) was first added to each well followed by 4 -h incubation at $37^{\circ} \mathrm{C}$. Finally, a lysis solution of $10 \% \mathrm{SDS}$ and $1 \mathrm{M} \mathrm{HCl}$ was added overnight in each well. For the readout, a multi-scanner microplate reader (Tecan GENios Microplate Reader, Männedorf, Switzerland) was used to measure the absorbance at $595 \mathrm{~nm}$. Each experiment was realized three times and each condition was performed in triplicates.

Flow cytometry. DLD1 cells were seeded onto 6-well plates at a density of 200,000 cells/well. After $24 \mathrm{~h}$, cells were treated for $24 \mathrm{~h}$ with $30 \mu \mathrm{M}$ AP or DMSO. Adherent cells were trypsinized and pooled together with non-adherent cells, rinsed with PBS and fixed with ice cold ethanol $70 \%$ for $2 \mathrm{~h}$ minimum. Cells were washed with PBS and stained for $30 \mathrm{~min}$ with a staining solution: PBS, $0.1 \%$ Triton X-100 (Sigma), $0.2 \mathrm{mg} / \mathrm{ml}$ RNAse A (Qiagen, Hilden, Germany) and $0.02 \mathrm{mg} / \mathrm{ml}$ propidium iodide (Sigma-Aldrich). Cells were analyzed by BD LSRFortessa cell analyzer (BD Biosciences, Heidelberg, Germany).

Sphere formation culture. Sphere formation medium was prepared as follows: DMEM-F12 with $1 \%$ penicillin/streptomycin, $10 \mathrm{ng} / \mathrm{ml}$ human recombinant $\beta \mathrm{FGF}, 20 \mathrm{ng} / \mathrm{ml}$ human recombinant EGF, $1 \%$ glutamine and B27 serum-free supplement $1 \mathrm{X}$ (all from Invitrogen) subsequently filtered with a 0.475- $\mu \mathrm{m}$ ultra Cruz syringe filter (Santa Cruz Biotechnology, Heidelberg, Germany). Parental cells were trypsinized, rinsed twice with PBS and cell counting was performed to seed 500 cells onto a 96-well ultra-low attachment plate (Corning $\mathrm{GmbH}$, Wiesbaden, Germany), each well containing $100 \mu \mathrm{l}$ of filtered sphere formation medium. Spheres were cultivated for 8 days and $100 \mu \mathrm{l}$ of media containing the additives ( $20 \mu \mathrm{M}$ AP, $100 \mathrm{ng} / \mathrm{ml}$ SP or DMSO) was added every 3 days. Sphere formation ability was determined under a microscope.

Reverse-phase protein array (RPPA). Cells were first treated with AP (20 or $40 \mu \mathrm{M})$, SP (100 ng/ml) or DMSO for $24 \mathrm{~h}$, washed with ice-cold PBS, denatured with a $1 \% \mathrm{SDS} / \beta$ mercaptoethanol lysis buffer solution and stored at $-80^{\circ} \mathrm{C}$. The Functional Proteomics RPPA Core Facility at MD Anderson Cancer Center analyzed the samples as previously described (23). In brief, probes were spotted on nitrocellulose slides and 172 different proteins were analyzed. Densitometry of these spots was performed and values normalized for protein loading. The values were then transformed to linear values, which were used for our calculations. The quantification was realized by calculating the ratios of the linear protein intensity values of the treated probes with the linear protein intensity values of their respective control probes.

Western blot analyses. LiM6 and DLD1 cells were first treated with AP $(30 \mu \mathrm{M})$ or DMSO for $24 \mathrm{~h}$, washed with ice-cold PBS and lysed with a lysis buffer $(0.5 \%$ Triton X-100, $1 \mathrm{mM}$ sodium orthovanadate, 1 protease inhibitor cocktail tablet in purified water). The protein concentration was determined by using the Bio-Rad protein assay (Bio-Rad Laboratories, Munich, Germany). For each condition, $20 \mu \mathrm{g}$ of proteins were loaded on $8-12 \%$ Tris-glycine gels (Invitrogen), separated by electrophoresis and electroblotted onto nitrocellulose membranes 
(Amersham Life Sciences, Buckinghamshire, UK). The membranes were then incubated for $2 \mathrm{~h}$ in a blocking solution (5\% BSA in TBS, $0.1 \%$ Tween-20), followed by an overnight incubation with primary antibodies at a 1:1,000 dilution against $\beta$-catenin, LRP5 (low-density lipoprotein receptor-related protein 5), MYC, PARP [poly(ADP-ribose) polymerase] and $\beta$-actin (all Cell Signaling Technologies, Danvers, MA, USA). Finally, the blots were washed with TBS-0.1\% Tween-20 and incubated for $1 \mathrm{~h}$ at room temperature with a peroxidaseconjugated goat anti-rabbit IgG antibody (Dako, Hamburg, Germany) at a dilution of 1:2,000. The detection was realized with an enhanced chemiluminescence reaction (ECL Prime Western blotting detection; Amersham Life Sciences) and $\beta$-actin served as a loading control.

Immunofluorescence. DLD1 cells were seeded onto 18-mm diameter cover slips (Thermo Scientific, Braunschweig, Germany) in a 12-well plates format (NUNC) at a density of 75,000 cells/well. Cells were treated with $30 \mu \mathrm{M} \mathrm{AP}, 70 \mathrm{nM} \mathrm{SP}$ or DMSO for $24 \mathrm{~h}$. Cells were then fixed with $4 \%$ paraformaldehyde for $15 \mathrm{~min}$, followed by a permeabilization step for $15 \mathrm{~min}$ with $0.15 \%$ Triton X-100 in PBS and a blocking step for $30 \mathrm{~min}$ with $1 \%$ BSA in PBS. The cells were then incubated overnight at $4^{\circ} \mathrm{C}$ with a rabbit primary antibody against $\beta$-catenin (Cell Signaling Technologies) diluted in blocking solution at 1:80. After multiple washing steps, cells were incubated in the dark with a goat anti-rabbit secondary antibody conjugated with Alexa Fluor 488 (Invitrogen) at a 1:200 dilution for $1 \mathrm{~h}$. Finally, cover slips were rinsed three times with PBS and mounted on microscope slides with Vectashield (Vector Laboratories, Burlingame, CA, USA) containing 4,6-diamidino-2-pheylindole (DAPI). The images were taken with a Olympus FluoView ${ }^{\mathrm{TM}}$ FV1000 confocal microscope.

$R N A$ isolation and RT-qPCR. Total RNA isolation was realized with TRI-reagent (Sigma-Aldrich) and $2 \mu \mathrm{g}$ were used for cDNA synthesis using random primers (Roche Diagnostics, Penzberg, Germany) and SuperScript II Reverse Transcriptase (Invitrogen). Each PCR reaction was made with $40 \mathrm{ng}$ of cDNA, $500 \mathrm{nM}$ of the primer pair and SoAdvanced ${ }^{\mathrm{TM}}$ Universal SYBR Green Supermix (Bio-Rad Laboratories) for a final volume of $20 \mu \mathrm{l}$. Thermal cycling was realized by the Mastercycler EP gradient S (Eppendorf, Hamburg, Germany) and consisted of 40 cycles with denaturation at $95^{\circ} \mathrm{C}$ for $15 \mathrm{sec}$, annealing at $55^{\circ} \mathrm{C}$ for $15 \mathrm{sec}$ and elongation at $72^{\circ} \mathrm{C}$ for $30 \mathrm{sec}$. All experimental conditions were assessed in duplicate. The primers used were as follows: CTNNB1 (NM_001904.2) forward, 5'-ACGTCCATGGGTGGGACA-3'; reverse, 5'-CTA GGATGTGAAGGGCTCCG-3'. AXIN2 (NM_004655.3) forward, 5'-TATCCAGTGATGCGCTGACG-3'; reverse, 5'-TGT TTCTTACTGCCCACACGAT-3'. MYC (NM_002467.3) forward, 5'-CACCACCAGCAGCGACTCT-3'; reverse, 5'-CAG ACTCTGACCTTTTGCCAGG-3'. FOXMI (NM_202002.2) forward, 5'-CTCCCGCAGCATCAAGCAA-3'; reverse, 5'-GCCAGGACGCTGATGGTCTC-3'.

TOP/FOP luciferase reporter assay. To assess luciferase activity we used the Dual-Luciferase Reporter Assay system (Promega, Madison, WI, USA). DLD1 and LiM6 cells were seeded in 24-well plates at a density of 35,000 cells/well for
24 h. For the transfection, we used FuGENE HD reagent (Promega) and $\alpha$ MEM medium. We co-transfected inducible Firefly luciferase expressing SuperTOP or SuperFOP vectors (AddGene plasmids nos. 12456 and 12457) and constitutively Renilla luciferase expressing normalization vector pRL-TK (Promega) at a ratio of 50:1. The latter vector was used as a control of transfection efficiency. Simultaneously, the cells were treated with different concentrations of AP as indicated or with DMSO. After $24 \mathrm{~h}$ of incubation total cell lysate was extracted using reporter lysis buffer (Promega). Total extract $(5 \mu 1), 25 \mu 1$ of Luciferase substrate LarII solution (Promega) and $25 \mu 1$ of Stop and Glow 1X solution (Promega) were used to assess Firefly and Renilla luciferase activities, respectively. Finally, Wnt activity was measured by the ratio of SuperTOP/SuperFOP luciferase activities each normalized to the respective Renilla luciferase activities. The results are displayed as relative light unit (RLU) and each experimental condition was assessed in triplicate.

Statistical analyses. Results are expressed as the mean \pm standard error of the mean (SEM). All statistical comparisons were made with a standard t-test and Mann-Whitney U test using biostatistics software from GraphPad Prism ${ }^{\circledR}$ (La Jolla, CA, USA). The criterion for significance was $p<0.05$ and $p<0.01$ for all comparisons.

\section{Results}

NK1R blocking leads to the regulation of specific proteins involved in different signaling pathways. We have recently described that upon NK1R blockage, growth of hepatoblastoma cells could be inhibited in vitro and in vivo (19). Here, we treated colon cancer cell lines LiM6 ( $\beta$-catenin mutation) (24) and DLD1 (APC mutation) (25) with increasing doses of AP for $48 \mathrm{~h}$ and then performed standard MTT assays. We observed a dose-dependent inhibition of cell growth in both cell lines (Fig. 1A).

In order to have a better understanding of the downstream molecular mechanisms responsible for these observed effects, we used reverse phase protein array (RPPA) analysis. We treated human CRC cells LiM6 and DLD1 with 20 or $40 \mu \mathrm{M}$ AP for $24 \mathrm{~h}$ and screened for changes in protein expression level of 172 proteins involved in multiple signaling pathways that are typically altered in cancer (23). Values from 20 and $40 \mu \mathrm{M}$ were put into relation to the control value of each cell line and the pathway molecule (Fig. 1B).

To scrutinize the regulation pattern in the two cell lines, we calculated the mean of the values relative to the control of 20 plus $40 \mu \mathrm{M}$ conditions for each molecule and displayed the results in Fig. 1C. The cut off values are specified in the figure legend. Only a small number of proteins exhibited an upregulation (red) or downregulation (green) common in the two cell lines. Interestingly, we found that important proteins associated with Wnt signaling were downregulated, including the Wnt target MYC and the $\beta$-catenin interacting factor FOXM1 (Fig. 1D). Taken together, these findings suggest inhibition of Wnt signaling through inhibition of NK1R. Of note, in these early experiments $\beta$-catenin, one key component of the Wnt signaling pathway, was not downregulated in DLD1 (Fig. 1C and D). 
A

LiM6
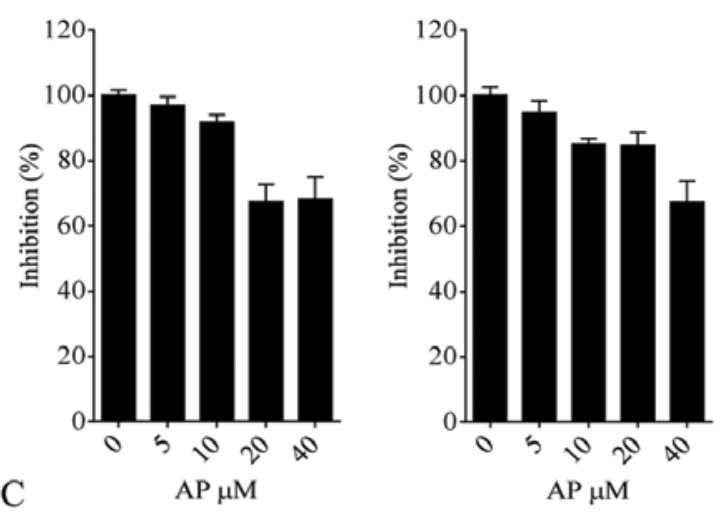

B

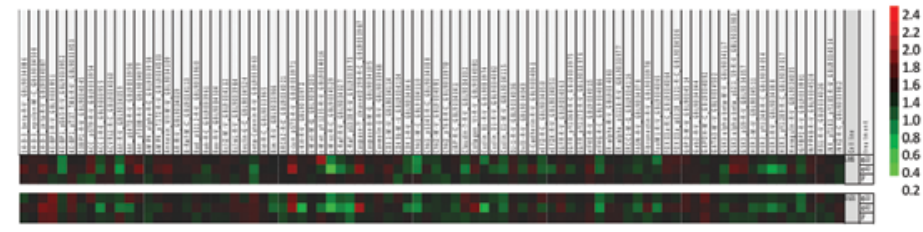

$\mathrm{D}$
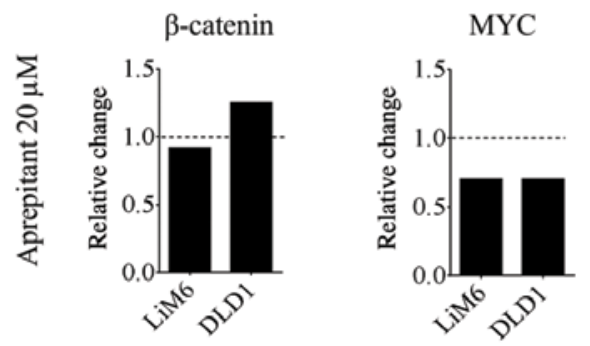

FOXM1
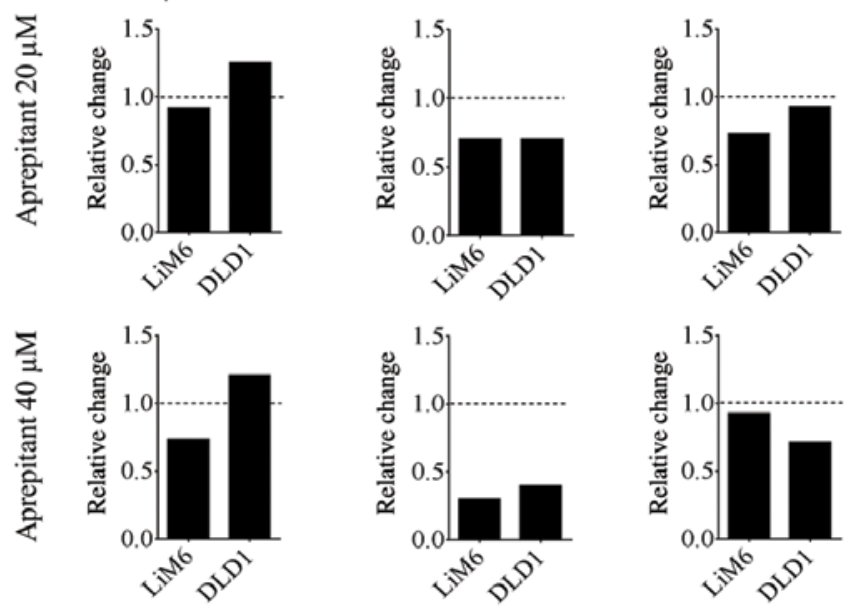

Figure 1. RPPA reveals multiple proteins being up and downregulated in colorectal cancer cells after AP treatment. (A) MTT assay in DLD1 and LiM6. Cells were treated with increasing concentration of AP or with DMSO (control) for $48 \mathrm{~h}$ to assess cell survival. (B) Cells were treated for $24 \mathrm{~h}$ with AP (20 or $40 \mu \mathrm{M}$ ), SP (70 $\mathrm{nM}$ ) or DMSO (control) and protein lysates were analyzed by RPPA. Heat map is shown representing an increased (red) or decreased signal (green) upon AP treatment normalized against the control. (C) The data are presented as the mean after AP treatment (20 and $40 \mu \mathrm{M}$ ) relative to control (DMSO) in DLD1 and LiM6 combined. The cut-offs were chosen according to the standard deviation of the values. In red, the selected proteins with a relative fold-change $\geq 1.163$ and in green, the selected proteins with a relative fold-change $\leq 0.836$ are shown. (D) Graphical representation of relative fold-changes of proteins associated with Wnt pathway ( $\beta$-catenin, MYC, FOXM1).

NKIR antagonism inhibits the AKT/mTOR pathway. Apart from Wnt signaling, we also found the AKT/mTOR pathway to be inhibited (Fig. 2, right panels) at the level of its downstream proteins phospho-4EBP1/2 and phospho-p70S6K (grey bars) compared to their total forms (black bars). Total AKT was also dose-dependently downregulated upon AP treatment in the two cell lines, whereas no clear trend could be extracted from its phospho-form at Thr308. In contrast, phosphorylation of AKT at Ser473 was upregulated (Fig. 2, left panels) along with its substrate PRAS40 at Thr246, a member and repressor of the TORC1 complex (26). These results indicate that apart from decreased Wnt signaling, inhibition of NK1R might lead to additional downregulation of the AKT/mTOR signaling pathway.

$N K 1 R$ antagonism induces apoptosis and a G2 arrest. It is known that NK1R antagonists induce apoptosis in human hepatoblastoma cancer cells and other cancers $(19,20)$. Therefore, as a next step, we investigated whether this held true in our setup. First, we screened our data obtained by RPPA for alterations in apoptosis markers. We found that cleaved caspase 7 and
phospho-c-Jun (Ser73) were upregulated upon AP treatment (Fig. 3A), two well known mediators of apoptosis $(27,28)$.

In order to characterize apoptosis in more detail, we treated DLD1 cells for $24 \mathrm{~h}$ with increasing concentration of AP and performed western blot analysis for PARP which executes the apoptotic process (29). We found a dose-dependent increase of cleaved PARP indicating an activation of apoptosis, whereas SP had no effect (Fig. 3B). Further, Her3 was found downregulated (Fig. 3A, right panels) and targeting this receptor in colorectal cancer cells has been described to induce a G2 arrest as well as apoptosis (30). We then analyzed the effect of AP on the expression of cell cycle regulators. In our RPPA data, we found cyclin B1 and CHK1 to be downregulated, whereas p53 was slightly upregulated (Fig. 3C). These proteins are known to regulate the G2-M transition (31). To investigate more in depth the growth inhibition observed in DLD1 and to confirm our RPPA data, we treated the cells for $24 \mathrm{~h}$ with $30 \mu \mathrm{M} \mathrm{AP}$ and analyzed the cell cycle profile by propidium iodide staining and FACS analysis. As shown in Fig. 3D, AP induced a G2 arrest (33\% compared with $26 \%$ for DMSO-treated cells, white bar) and an increase of cells in the 
AKT (S473)
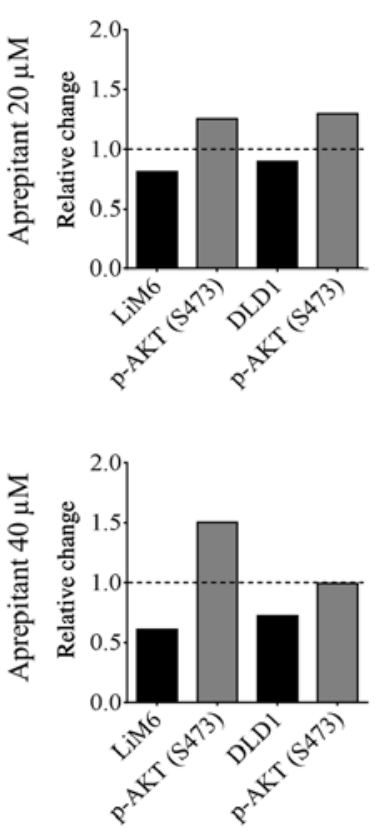

AKT (T308)
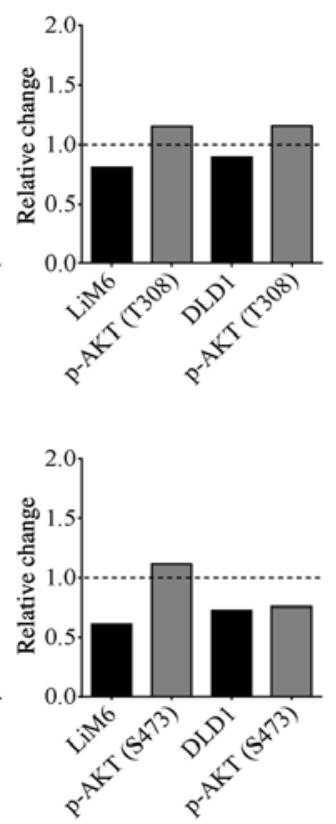

PRAS40 (T246)
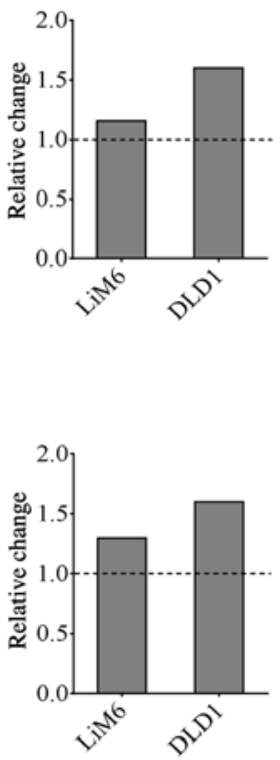

p70S6K

$4 \mathrm{E}-\mathrm{BP} 1 / 2$
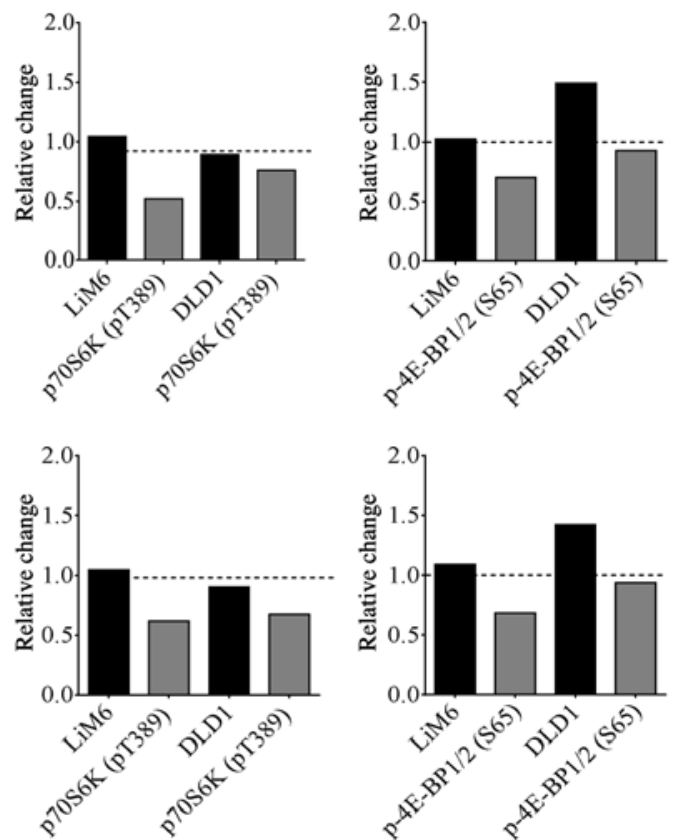

Figure 2. RPPA reveals an inhibition of AKT/mTOR pathway in colorectal cancer cells. Relative fold-changes of proteins involved in AKT/mTOR pathway. Cells were treated with 20 or $40 \mu \mathrm{M}$ AP, values were put into relation with the control (DMSO) and normalized to 1. For AKT (S473), AKT (T308), PRAS (T246), p70S6K and 4E-BP1 the phosphorylated proteins are shown in grey bars and the total proteins in black bars.

A

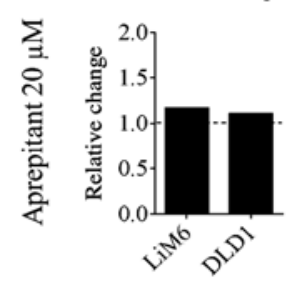

C

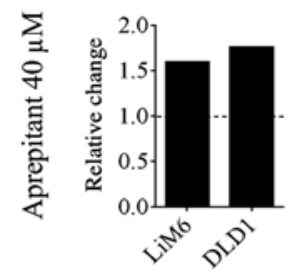

Cyclin B1
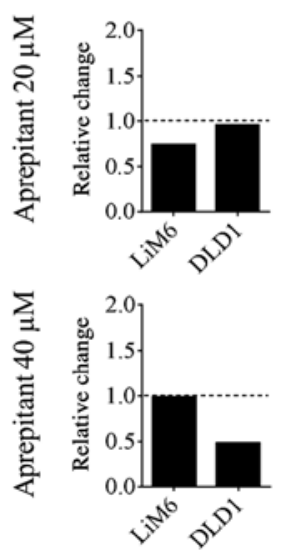

c-Jun $(\operatorname{ser} 73)$
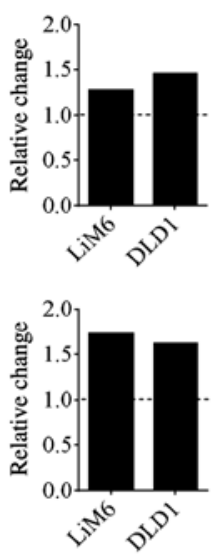

CHK1
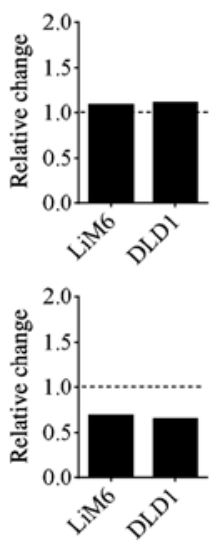

Her3
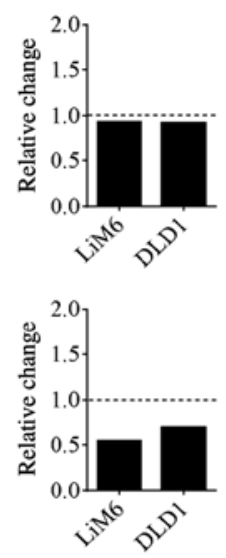

p53
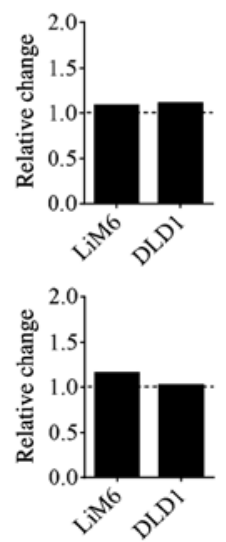

B
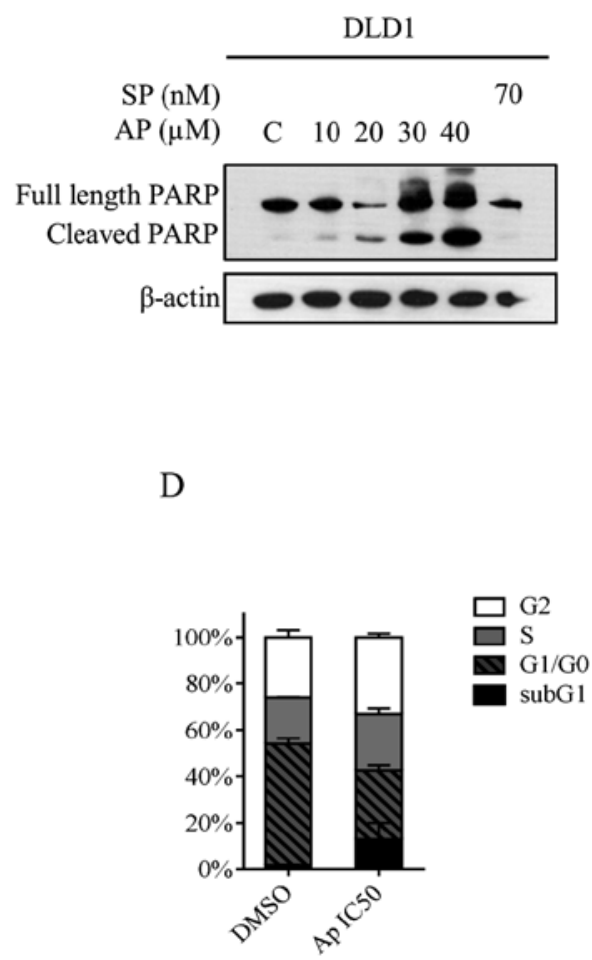

Figure 3. AP induces a G2 arrest and apoptosis in colorectal cancer cells. (A) Relative fold-change of selected up and downregulated proteins involved in apoptosis or (C) in cell cycle regulation are shown. (B) Western blot analysis of total and cleaved PARP after treatment with increasing doses of AP for $24 \mathrm{~h}$. (D) Analysis of the cell cycle by flow cytometry in DLD1. Cells were treated with $30 \mu \mathrm{M}$ AP or DMSO for $24 \mathrm{~h}$ and stained with propidium iodide. The data are presented as the percentage of cells at different stages: subG1 (black), G1/G0 (black and grey), S (grey) or G2 (white). Values are shown as percentage. 


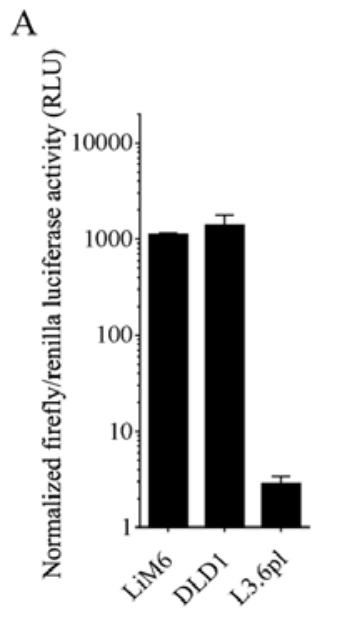

$\mathrm{D}$
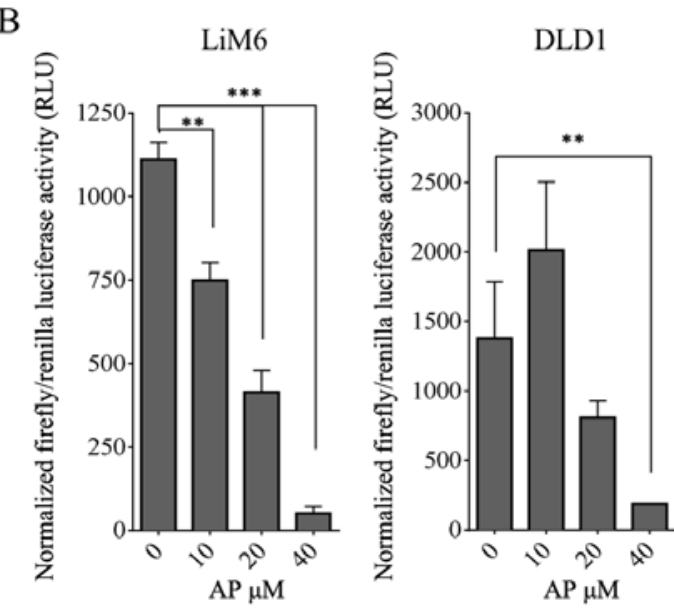

$\mathrm{E}$

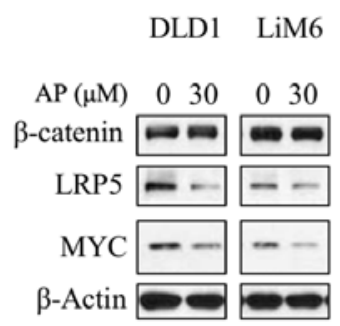

$\mathrm{C}$

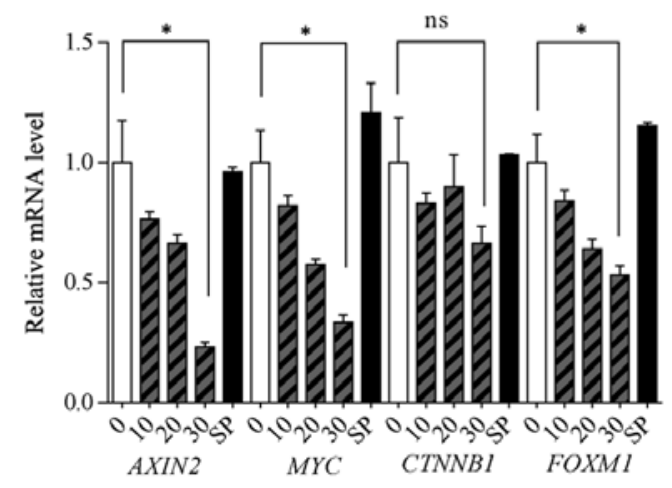

$\mathrm{F}$

DLD1

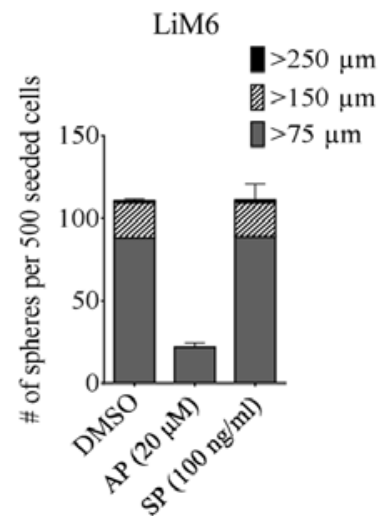

Figure 4. AP induces a robust inhibition of Wnt activity and promotes membrane-bound $\beta$-catenin in colorectal cancer cells. (A) Baseline Wnt activity was assessed using the SuperTOP/FOP reporter system. Cells were transfected with SuperTOP (non-mutated TCF/LEF binding sites) or SuperFOP (mutated TCF/LEF binding sites) for $24 \mathrm{~h}$. Results are presented by the ratio of SuperTOP/SuperFOP Firefly luciferase and normalized to Renilla luciferase. (B) Ratio of SuperTOP/FOP (A) after $24 \mathrm{~h}$ of treatment with different concentrations of AP (10, 20 or $40 \mu \mathrm{M})$ or DMSO (0) in LiM6 and DLD1. (C) qRT-PCR of Wnt target genes (AXIN2, MYC, CTNNB1) and Wnt-associated gene (FOXM1). Cells were treated with increasing doses of AP $(10,20$ or $30 \mu \mathrm{M}$, gray and black columns), SP (black columns) or DMSO (white columns) for $24 \mathrm{~h}$. (D) Immunofluorescent staining of $\beta$-catenin (green) in DLD1 and nuclear staining with DAPI (blue). Cells were treated with $30 \mu \mathrm{M}$ AP, $70 \mathrm{nM}$ SP or DMSO for $24 \mathrm{~h}$. The staining was examined by confocal microscopy. (E) Selected proteins of the Wnt pathway were validated by western blot analysis ( $\beta$-catenin, LRP5, MYC). Cells were treated with $30 \mu \mathrm{M}$ AP or DMSO $(0)$ for $24 \mathrm{~h}$ followed by extraction of total cell lysates. (F) Sphere-forming assays (SFA) in DLD1 and LiM6 with simultaneous treatment with AP or SP. Shown are the number of spheres per 500 seeded cells in an ultra-low attachment 96-well plate after 10 days. Sphere sizes were evaluated and categorized as follows: $>250 \mu \mathrm{m}$ (black), $>150 \mu \mathrm{m}$ (black and grey) and $>75 \mu \mathrm{m}$ (grey) $\mathrm{n}=3$.

subG1 (12.7\% compared with $1.63 \%$ for DMSO-treated cells, black bar) phase indicating either late apoptosis or necrosis.

NK1R antagonism inhibits the canonical Wnt signaling. After analyzing the RPPA results above, we found strong evidence that AP might induce a reduction of Wnt activity. To validate this hypothesis, we then carried out Super TOP/FOP (STF) assays in non-treated cells to assess baseline Wnt activity in DLD1 and LiM6 cells and compared their activity to the pancreatic cancer cell line L3.6pl, a cell line known to express little Wnt. As expected, we found that both colon cancer cell lines displayed high levels of Wnt activity with a 500-fold increased activity compared to L3.6pl (Fig. 4A).

Next, we treated the colon cancer cells with increasing doses of AP. After a one-time treatment, we discovered a robust inhibition of Wnt activity in both colorectal cell lines (Fig. 4B). These results were corroborated by qRT-PCR data in DLD1. The Wnt target gene MYC was dose-dependently downregulated upon AP treatment along with AXIN2 and FOXMI (Fig. 4C). Intriguingly, in contrast to the RPPA data,
CTNNB1 was also downregulated, although the trend was not as convincing as observed in the other genes. Thus, instead of targeting $\beta$-catenin for degradation, AP might induce a decrease of Wnt activity by sequestrating it away from the nucleus resulting in decreased TCF/ $\beta$-catenin activity, ultimately leading to a lower Wnt target gene expression.

To further investigate this hypothesis and the downregulation of the $\beta$-catenin/Wnt signaling pathway following AP treatment, we cultured cells with $30 \mu \mathrm{M}$ AP or $70 \mathrm{nM}$ SP for $24 \mathrm{~h}$, stained them for $\beta$-catenin and analyzed them with confocal microscopy. At the membrane, $\beta$-catenin is required for cell adhesion where it complexes with E-cadherin. Consequently, despite being protected from degradation, $\beta$-catenin is not available for transducing a signal to the nucleus in this state (32). We observed a strong accumulation of membrane-bound $\beta$-catenin upon AP treatment (Fig. 4D). However, SP stimulation did not seem to affect $\beta$-catenin/Wnt signaling when compared to the control.

Finally, we treated the cells with $30 \mu \mathrm{M}$ AP and performed western blot analysis. We found that the Wnt co-receptor LRP5 
A
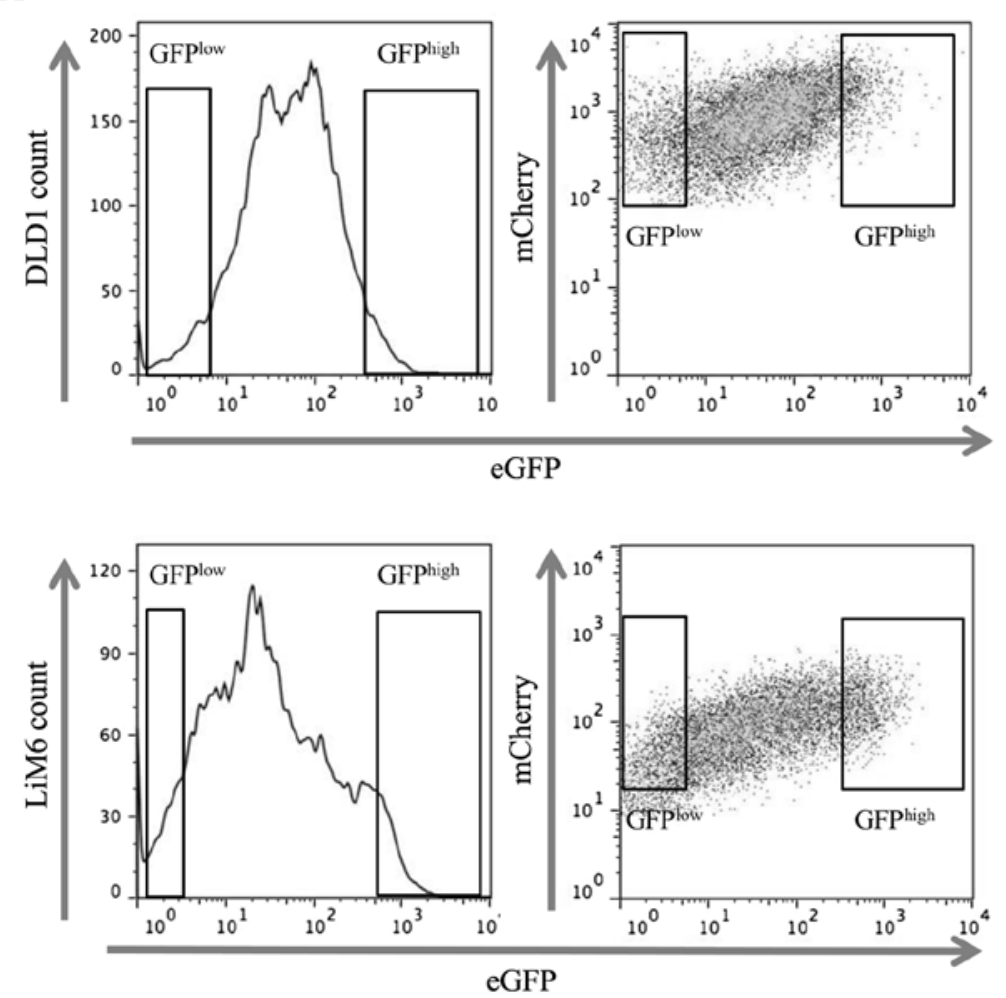

B
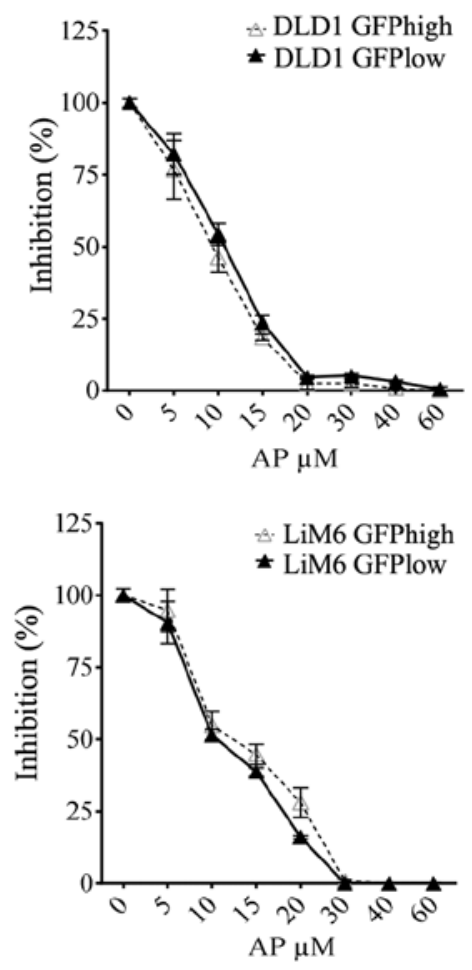

Figure 5. AP affects the cell viability of GFP/Wnt ${ }^{\text {high }}$ and GFP/Wnt ${ }^{\text {low }}$ cells to the same degree. (A) CRC cells were transduced with the lentiviral construct 7xTCF-eGFP/SV40-mCherry (7TGC). The 5\% GFPhigh and 5\% GFP low cells were sorted via FACS and used for further evaluation. Shown are representative FACS plots with DLD1 in the upper panel and LiM6 in the lower panel. The left panels show cell counts on the y-axis and eGFP intensities on the x-axis; the right panels show mCherry intensities on the $y$-axis and eGFP intensities on the $\mathrm{x}$-axis. (B) GFP ${ }^{\text {high }}$ or GFPlow cells from (A) were treated for $24 \mathrm{~h}$ with increasing doses of AP. Subsequently, MTT cell viability assays were carried out and relative cell viability comparedto DMSO treated cells is shown ( $=3$ ).

as well as the Wnt target MYC had markedly lower expression upon AP treatment compared to the respective control. In the same experiment, we analyzed the expression of $\beta$-catenin following AP treatment. Similar to the RPPA data, we did not observe any striking changes of total $\beta$-catenin in whole cell lysates of LiM6 or DLD1 (Fig. 4E). Taken together, these data demonstrate that targeting the NK1R in colon cancer cell lines markedly reduces Wnt-signaling.

In a next step, we investigated whether this held true not only in differentiated colon cancer cell lines, but also in colon cancer stem cells (CSCs). We grew colorectal CSC-like cells as described (33) and assessed sphere formation ability (SFA) in both DLD1 and LiM6 (Fig. 4F). Upon AP treatment, we discovered a striking decrease, both in sphere number and size. Interestingly, activation of the SP/NK1R system with recombinant SP significantly increased the SFA of DLD1 CSCs in number and size, whereas in LiM6, we were not able to find differences between SP-stimulated cells and control (Fig. 4F).

Taken together, these experiments show that inhibition of the SP/NK1R system with AP decreased the canonical Wnt signaling in colorectal cancer cells, likely by arresting $\beta$-catenin in its membrane-bound localization. Also, inhibition of the SP/NK1R system inhibited the growth of anoikisresistant CSC-like colorectal spheres.

The inactivation of the $\beta$-catenin/Wnt pathway is independent of the initial Wnt baseline activity. In order to better understand the inhibitory effect on Wnt signaling, we sought to investigate whether differences exist regarding the response rate to AP treatment within cell populations that are constitutively active regarding the $\beta$-catenin/Wnt pathway. Such cells with high constitutive Wnt activation have been described to have higher stemness properties (6). To make our findings comparable to those from Vermeulen et al, we transduced the colorectal cell lines LiM6 and DLD1 with a 7xTCF-eGFP/mCherry (7TGC) lentiviral construct (34) allowing us to separate cells with different Wnt activities by single cell sorting via FACS (Fig. 5A). Wnt activity in cells with the same level of mCherry intensity then correlates with their GFP intensity. After separation, we independently cultured $\mathrm{Wnt}^{\text {high }}$ and $\mathrm{Wnt}^{\text {low }}$ expressing cells and subsequently treated these cells with increasing doses of AP for 48 h (Fig. 5B). Interestingly, we did not observe a difference in cell survival for any of the doses tested between cells with high or low Wnt activity. These findings suggest that the apoptosis-inducing property of NK1R targeting is maintained even in cells with supposedly increased cancer stemness potential ( $\mathrm{Wnt}^{\text {high }}$ ). Furthermore, our data indicate that in a particular cell line or population, the observed inhibitory effects caused by NK1R antagonism are independent of the initial Wnt baseline activation.

\section{Discussion}

It is well established that aberrant activation of Wnt signaling is a key event in colorectal tumorigenesis. Therefore, recent efforts have focused on developing new molecules that block 
this pathway. For this purpose, the use of antibody-based therapy, small molecules (35) or other direct and indirect inhibitors (36) have all been studied in depth, but to date no component exists that has succeeded at a clinical level. In this study, we found that aprepitant (AP), an NK1R antagonist currently approved by the FDA for the treatment of chemotherapy-induced nausea and vomiting, triggered a robust inhibition of $\mathrm{Wnt} / \beta$-catenin signaling activity and a growth inhibition in colorectal cancer cells. Although $\beta$-catenin was not clearly downregulated at the protein level or mRNA level, we found that AP induced an accumulation in its membrane-bound state, where it cannot execute its function as a transcriptional co-activator. Consequently, we found a significant inhibition of $\mathrm{Wnt} / \beta$-catenin activity as measured by the luciferase reporter assay.

The cell lines used in our study were DLD1 and LiM6, which harbor $A P C$ and $\beta$-catenin mutations, respectively. Consequently, the molecules upstream of the destruction complex, the latter being composed of AXIN, adenomatous polyposis coli (APC), glycogen synthase kinase $3 \beta$ (GSK3 $\beta$ ), casein kinase 1 (CK1) and protein phosphatase 2A (PP2A) (37), should not be needed for pathway activation/modulation. Taking this into account, it appears that AP acts either at the level of $\beta$-catenin or downstream of it. Therefore, we hypothesized that the inhibition of Wnt activity was due either to the loss of $\beta$-catenin translocation into the nucleus or by inhibition of $\beta$-catenin binding to the transcription factors TCF/LEF. In this sense, it has been demonstrated that $\beta$-catenin activation directly correlates with FOXM1 and nestin expression, a stem cell marker (38). Moreover, it has been suggested that FOXM1 might be crucial for $\mathrm{Wnt} / \beta$-catenin activity. This could be demonstrated in FOXM1 knockout mice where Wnt/ $\beta$-catenin

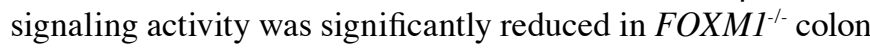
tumors (39). In accordance with these data, in our study we found FOXM1 to be significantly downregulated following treatment with AP.

We performed immunofluorescence stainings for $\beta$-catenin to detect its cellular localization following NK1R inhibition. Intriguingly, we discovered strong accumulation of membrane-bound $\beta$-catenin reflecting an inhibition of Wnt activity. As mentioned above, one explanation for this could be downregulation of FOXM1 expression.

Another, perhaps more grounded hypothesis is that AP induces an arrest in its membrane-bound localization, which then subsequently inhibits its function as a transcriptional co-activator by binding to TCF/LEF. Indeed, while free cytosolic $\beta$-catenin is normally required for signal transduction, membrane-bound $\beta$-catenin is sequestrated for cell adhesion and consequently protected from degradation. Unfortunately, our data do not permit us to extrapolate by which mechanisms $\beta$-catenin accumulates at the membrane. A possible explanation suggested by previous reports (40) is that E-cadherin phosphorylation promotes cell adhesion by increasing the E-cadherin $/ \beta$ catenin affinity. Particularly the cytoplasmic domain of E-cadherin contains several phosphorylation sites for casein kinase II (CKII) and GSK3 $\beta$.

A third explanation for our findings involves a mechanism consisting in a direct or indirect inhibition of the $\mathrm{TCF} / \beta$-catenin complex by AP. Previous reports have shown that several small molecules specifically disrupt the TCF7L $2 / \beta$-catenin complex and thus trigger growth inhibition in colorectal cells in vitro and in vivo $(41,42)$.

The described mechanism of inhibition downstream of $\beta$-catenin is particularly interesting, because this form of targeting Wnt signaling could potentially avoid some side effects that have been claimed to be unique to inhibition upstream of $\beta$-catenin. For example, with the inhibition downstream of $\beta$-catenin, physiologic complex formation such as E-cadherin/ $\beta$-catenin, which ensures proper cell adhesion in normal cells, is not disrupted. Nevertheless, inhibition downstream of $\beta$-catenin will nonetheless target Wnt target genes, which are the main actors of tumorigenesis. As suspected, we discovered a downregulation at the mRNA level and/or protein level of several important target genes such as MYC, AXIN2 and $C C N D 1$.

Another interesting finding of our study was the fact that treatment with AP resulted in growth inhibition of cells independent of their constitutional Wnt activity. For example, we observed the same therapeutic effect after separating colon cancer cells in $\mathrm{Wnt}^{\text {high }}$ and $\mathrm{Wnt}^{\text {low }}$ expressing cells. Furthermore, when we treated the pancreatic cancer cell line L3.6pl, a cell line known to express minimal Wnt, we also observed a strong growth inhibition (Ilmer et al, unpublished data). This can potentially have several explanations. Given the critical role of Wnt signaling for any cancer cell, it is likely that abrogation of Wnt signaling will have detrimental effects on the cell, regardless of its constitutional Wnt activity. On the other hand, these results could be explained with the fact that, most likely, Wnt inhibition is not the only mechanism by which AP inhibits cancer cells. The latter would be the more encouraging explanation, since it is known that cancer cells have enormous potential to escape anticancer agents, especially if they rely on one particular pathway. Indeed, in our study, along with inhibition of Wnt signaling, we found the AKT/mTOR signaling pathway to be downregulated significantly.

AKT/mTOR signaling pathway is known to be activated in cancer cells and is involved in tumorigenesis. Thus, the inhibition of AKT/mTOR by itself is undergoing thorough investigation as a promising candidate for future anticancer strategies. Interestingly, in accordance to our data, mTOR inhibition following NK1R blockage has been described for other cancers (43). Given that in our study we did not only see robust inhibition of AKT/mTOR, but also significant inhibition on Wnt signaling, we wonder whether the two pathways counter-affect each other and if a defined, inverse cross-link is activated between the two following AP treatment. Crosslinks between the AKT/mTOR and Wnt pathways have been described. For example, in the absence of $\beta$-catenin, GSK $3 \beta$ can be inhibited to activate mTOR. Inversely, Wnt signaling can activate TSC2, which negatively regulates mTOR (44). In our study, we found mainly a downregulation of total AKT and its downstream target $\mathrm{p} 70 \mathrm{~S} 6 \mathrm{~K}, 4 \mathrm{E}-\mathrm{BP} 1 / 2$ indicating an overall pathway inhibition. Unlike our expectation, p-AKT (Ser473) and PRAS40 (Thr246) were found to be upregulated. This phenomenon has already been described following the inhibition of the mTORC1 and is believed to be mediated through mTORC2 complex. It has been suggested that upregulation of p-AKT at Ser473 could be responsible for the development 
of drug resistance (45). Unfortunately, at this point we cannot extract from our data insight into whether treatment with AP activates a defined cross-link between the two pathways or if the downstream effects are completely independent from one another.

Taken together, our results indicate that AP inhibits cell growth of colorectal as well as CSC-like cancer cells. Upon NK1R inhibition, we found two pivotal cancer pathways to be repressed: Wnt and AKT/mTOR. Therefore, we here highlight for the first time insight into the intracellular mechanisms triggered by AP treatment in colorectal cancer cells. These findings could be of great importance for the generation of an effective anticancer therapy against colorectal and other cancers.

\section{Acknowledgements}

Michael Berger and Matthias Ilmer were supported by postdoctoral stipends of the German Academic Exchange Program (DAAD). Michael Berger was additionally funded by the Friedrich-Baur foundation Munich, Münchener Medizinische Wochenschrift, as well as the Förderung für Forschung und Lehre of LMU University. This study was supported in part by grants of the Alliance of Cardiovascular Researchers to Eckhard Alt. Roland Kappler obtained funding from the Bettina Bräu foundation, Munich, and the GänseblümchenVoerde foundation, Voerde.

\section{References}

1. Boyle P and Langman JS: ABC of colorectal cancer: Epidemiology. BMJ 321: 805-808, 2000

2. Fearon ER: Molecular genetics of colorectal cancer. Annu Rev Pathol 6: 479-507, 2011.

3. Fearnhead NS, Britton MP and Bodmer WF: The ABC of APC Hum Mol Genet 10: 721-733, 2001.

4. Giles RH, van Es JH and Clevers H: Caught up in a Wnt storm: Wnt signaling in cancer. Biochim Biophys Acta 1653: 1-24, 2003

5. Fodde R, Smits R and Clevers H: APC, signal transduction and genetic instability in colorectal cancer. Nat Rev Cancer 1: 55-67, 2001 .

6. Vermeulen L, De Sousa E Melo F, van der Heijden M, Cameron K, de Jong JH, Borovski T, Tuynman JB, Todaro M, Merz $\mathrm{C}$, Rodermond $\mathrm{H}$, et al: Wnt activity defines colon cancer stem cells and is regulated by the microenvironment. Nat Cell Biol 12: 468-476, 2010.

7. Ilmer M, Boiles AR, Regel I, Yokoi K, Michalski CW, Wistuba II, Rodriguez J, Alt E and Vykoukal J: RSPO2 enhances canonical Wnt signaling to confer stemness-associated traits to susceptible pancreatic cancer cells. Cancer Res 75: 1883-1896, 2015.

8. Prud'homme GJ: Cancer stem cells and novel targets for antitumor strategies. Curr Pharm Des 18: 2838-2849, 2012.

9. Reya T, Morrison SJ, Clarke MF and Weissman IL: Stem cells, cancer, and cancer stem cells. Nature 414: 105-111, 2001.

10. Nagler C, Zänker KS and Dittmar T: Cell fusion, drug resistance and recurrence CSCs. Adv Exp Med Biol 714: 173-182, 2011.

11. Quartara L and Maggi CA: The tachykinin NK1 receptor Part II: Distribution and pathophysiological roles. Neuropeptides 32: 1-49, 1998.

12. Hökfelt T, Pernow B and Wahren J: Substance P: A pioneer amongst neuropeptides. J Intern Med 249: 27-40, 2001.

13. Varty GB, Cohen-Williams ME and Hunter JC: The antidepressant-like effects of neurokinin NK1 receptor antagonists in a gerbil tail suspension test. Behav Pharmacol 14: 87-95, 2003.

14. Rosso M, Muñoz M and Berger M: The role of neurokinin-1 receptor in the microenvironment of inflammation and cancer. Sci World J 2012: 381434, 2012.

15. Muñoz M and Coveñas R: Neurokinin-1 receptor: A new promising target in the treatment of cancer. Discov Med 10: 305-313, 2010 .
16. Muñoz M, Rosso $M$ and Coveñas R: A new frontier in the treatment of cancer: NK-1 receptor antagonists. Curr Med Chem 17: 504-516, 2010.

17. Kramer MS1, Winokur A, Kelsey J, Preskorn SH, Rothschild AJ, Snavely D, Ghosh K, Ball WA, Reines SA, Munjack D, et al: Demonstration of the efficacy and safety of a novel substance P (NK1) receptor antagonist in major depression. Neuropsychopharmacology 29: 385-392, 2004.

18. Kramer MS, Cutler N, Feighner J, Shrivastava R, Carman J, Sramek JJ, Reines SA, Liu G, Snavely D, Wyatt-Knowles E, et al: Distinct mechanism for antidepressant activity by blockade of central substance P receptors. Science 281: 1640-1645, 1998.

19. Berger M, Neth O, Ilmer M, Garnier A, Salinas-Martín MV, de Agustín Asencio JC, von Schweinitz D, Kappler R and Muñoz M: Hepatoblastoma cells express truncated neurokinin-1 receptor and can be growth inhibited by aprepitant in vitro and in vivo. J Hepatol 60: 985-994, 2014.

20. Muñoz M, González-Ortega A, Salinas-Martín MV, Carranza A, Garcia-Recio S, Almendro V and Coveñas R: The neurokinin-1 receptor antagonist aprepitant is a promising candidate for the treatment of breast cancer. Int J Oncol 45: 1658-1672, 2014.

21. Muñoz M and Rosso M: The NK-1 receptor antagonist aprepitant as a broad spectrum antitumor drug. Invest New Drugs 28: 187-193, 2010.

22. Bresalier RS, Niv Y, Byrd JC, Duh QY, Toribara NW, Rockwell RW, Dahiya R and Kim YS: Mucin production by human colonic carcinoma cells correlates with their metastatic potential in animal models of colon cancer metastasis. J Clin Invest 87: 1037-1045, 1991.

23. Hennessy BT, Lu Y, Gonzalez-Angulo AM, Carey MS, Myhre S, Ju Z, Davies MA, Liu W, Coombes K, Meric-Bernstam F, et al: A technical assessment of the utility of reverse phase protein arrays for the study of the functional proteome in non-microdissected human breast cancers. Clin Proteomics 6: 129-151, 2010.

24. Brabletz T, Jung A, Reu S, Porzner M, Hlubek F, KunzSchughart LA, Knuechel R and Kirchner T: Variable beta-catenin expression in colorectal cancers indicates tumor progression driven by the tumor environment. Proc Natl Acad Sci USA 98: 10356-10361, 2001

25. Yang J, Zhang W, Evans PM, Chen X, He X and Liu C: Adenomatous polyposis coli (APC) differentially regulates betacatenin phosphorylation and ubiquitination in colon cancer cells. J Biol Chem 281: 17751-17757, 2006.

26. Wiza C, Nascimento EB and Ouwens DM: Role of PRAS40 in Akt and mTOR signaling in health and disease. Am J Physiol Endocrinol Metab 302: E1453-E1460, 2012.

27. Bossy-Wetzel E, Bakiri L and Yaniv M: Induction of apoptosis by the transcription factor c-Jun. EMBO J 16: 1695-1709, 1997.

28. Leppä S and Bohmann D: Diverse functions of JNK signaling and C-Jun in stress response and apoptosis. Oncogene 18: 6158-6162, 1999.

29. Lazebnik YA, Kaufmann SH, Desnoyers S, Poirier GG and Earnshaw WC: Cleavage of poly(ADP-ribose) polymerase by a proteinase with properties like ICE. Nature 371: 346-347, 1994.

30. Gespach C: Increasing potential of HER3 signaling in colon cancer progression and therapy. Clin Cancer Res 18: 917-919, 2012.

31. Taylor WR and Stark GR: Regulation of the G2/M transition by p53. Oncogene 20: 1803-1815, 2001.

32. Orsulic S, Huber O, Aberle H, Arnold S and Kemler R: E-cadherin binding prevents beta-catenin nuclear localization and beta-catenin/LEF-1-mediated transactivation. J Cell Sci 112: 1237-1245, 1999.

33. Lonardo E, Hermann PC, Mueller MT, Huber S, Balic A, Miranda-Lorenzo I, Zagorac S, Alcala S, Rodriguez-Arabaolaza I, Ramirez JC, et al: Nodal/Activin signaling drives self-renewal and tumorigenicity of pancreatic cancer stem cells and provides a target for combined drug therapy. Cell Stem Cell 9: 433-446, 2011.

34. Fuerer $\mathrm{C}$ and Nusse R: Lentiviral vectors to probe and manipulate the Wnt signaling pathway. PLoS One 5: e9370, 2010.

35. Lepourcelet M, Chen YN, France DS, Wang H, Crews P, Petersen F, Bruseo C, Wood AW and Shivdasani RA: Smallmolecule antagonists of the oncogenic Tcf/beta-catenin protein complex. Cancer Cell 5: 91-102, 2004.

36. Moon RT, Kohn AD, De Ferrari GV and Kaykas A: WNT and beta-catenin signalling: Diseases and therapies. Nat Rev Genet 5: 691-701, 2004.

37. Klaus A and Birchmeier W: Wnt signalling and its impact on development and cancer. Nat Rev Cancer 8: 387-398, 2008. 
38. Gong A and Huang S: FoxM1 and Wnt $/ \beta$-catenin signaling in glioma stem cells. Cancer Res 72: 5658-5662, 2012.

39. Yoshida Y, Wang IC, Yoder HM, Davidson NO and Costa RH: The forkhead box M1 transcription factor contributes to the development and growth of mouse colorectal cancer. Gastroenterology 132: 1420-1431, 2007.

40. Lickert H, Bauer A, Kemler R and Stappert J: Casein kinase II phosphorylation of E-cadherin increases E-cadherin/betacatenin interaction and strengthens cell-cell adhesion. J Biol Chem 275: 5090-5095, 2000.

41. Gonsalves FC, Klein K, Carson BB, Katz S, Ekas LA, Evans S, Nagourney R, Cardozo T, Brown AM and DasGupta R: An RNAi-based chemical genetic screen identifies three smallmolecule inhibitors of the Wnt/wingless signaling pathway. Proc Natl Acad Sci USA 108: 5954-5963, 2011.

42. Tian W, Han X, Yan M, Xu Y, Duggineni S, Lin N, Luo G, Li YM, Han X, Huang Z, et al: Structure-based discovery of a novel inhibitor targeting the $\beta$-catenin/Tcf4 interaction. Biochemistry 51: 724-731, 2012.
43. Mayordomo C, García-Recio S, Ametller E, FernándezNogueira P, Pastor-Arroyo EM, Vinyals L, Casas I, Gascón P and Almendro V: Targeting of substance $P$ induces cancer cell death and decreases the steady state of EGFR and Her2. J Cell Physiol 227: 1358-1366, 2012

44. Inoki $\mathrm{K}$, Ouyang $\mathrm{H}$, Zhu $\mathrm{T}$, Lindvall $\mathrm{C}$, Wang $\mathrm{Y}$, Zhang $\mathrm{X}$, Yang Q, Bennett C, Harada Y, Stankunas K, et al: TSC2 integrates Wnt and energy signals via a coordinated phosphorylation by AMPK and GSK 3 to regulate cell growth. Cell 126: 955-968, 2006.

45. Breuleux M, Klopfenstein M, Stephan C, Doughty CA, Barys L, Maira SM, Kwiatkowski D and Lane HA: Increased AKT S473 phosphorylation after mTORC1 inhibition is rictor dependent and does not predict tumor cell response to PI3K/mTOR inhibition. Mol Cancer Ther 8: 742-753, 2009. 